\title{
Cross-modal compatibility effects with visual-spatial and auditory-verbal stimulus and response sets
}

\author{
ROBERT W. PROCTOR, ADDIE DUTTA, and PAUL L. KELLY \\ Purdue University, West Lafayette, Indiana \\ and \\ DANIEL J. WEEKS \\ Simon Fraser University, Burnaby, British Columbia, Canada
}

\begin{abstract}
Within the visual-spatial and auditory-verbal modalities, reaction times to a stimulus have been shown to be faster if salient features of the stimulus and response sets correspond than if they do not. Accounts that attribute such stimulus-response compatibility effects to general translation processes predict that similar effects should occur for cross-modal stimulus and response sets. To test this prediction, three experiments were conducted examining four-choice reactions with (1) visual spatial-location stimuli assigned to speech responses, (2) speech stimuli assigned to keypress responses, and (3) symbolic visual stimuli assigned to speech responses. In all the experiments, responses were faster when correpondence between salient features of the stimulus and response sets was maintained, demonstrating that similar principles of translation operate both within and across modalities.
\end{abstract}

Performance of a perceptual-motor task requires that perceptual information be translated into action. The speed and accuracy with which many tasks can be performed is primarily a function of the difficulty of the required translation (Welford, 1976). Several factors, including the number of possible stimuli, the relation between the stimulus set and the action class, and the amount of practice, influence the difficulty of translation.

One of the most important factors affecting translation is the manner in which stimuli are assigned to responses. The effects that arise from different assignments of stimuli to responses, called stimulus-response (S-R) compatibility effects, have provided evidence about the workings of translation processes (Hasbroucq, Guiard, \& Ottomani, 1990; Proctor \& Reeve, 1990). In the majority of S-R compatibility studies, the stimulus set, the response set, or both, have a spatial feature. Research with these stimulus and response sets has shown that performance often is mediated by relative spatial codes, rather than by absolute spatial locations or the effectors used for responding (Umiltà \& Nicoletti, 1990). That is, it appears to be the mental representation of the sets that determines performance.

\footnotetext{
We would like to thank Bridget Robinson for preliminary discussions of this topic that contributed to the development of this project. The manuscript also benefited from comments on an earlier version provided by James Cutting, Albrecht Inhoff, Lester Krueger, Claire Michaels, Arty Samuel, and an anonymous reviewer. D.J.W. was supported by the Natural Sciences Engineering and Research Council of Canada. Reprint requests should be sent to R. W. Proctor, Psychology Department, Purdue University, 1364 Psychology Building, Rm. 3150, West Lafayette, IN 47907-1364.
}

More generally, the efficiency of translation processes has been proposed to follow a salient-features coding principle (Proctor, Reeve, \& Weeks, 1990; Reeve \& Proctor, 1990), according to which translation between stimuli and responses is faster when salient features of the stimulus and response sets correspond than when they do not. Results consistent with this principle are evident in a four-choice spatial-precuing task (Cauraugh \& Horrell, 1989; Miller, 1982; Reeve \& Proctor, 1984). In this task, the stimuli are four visual locations arranged in a row. The stimulus locations are assigned directly to four response keys, also arranged in a row, on which the index and middle fingers of each hand are placed. When two of the locations are precued prior to presentation of the imperative stimulus, a pattern of differential precuing benefits is obtained. Precuing is more beneficial if the two leftmost or two rightmost locations are cued than if other pairs of locations are cued. When the left/right spatial distinction is dissociated from the anatomical distinction between the left and right hands by manipulating hand placement, the same pattern of differential precuing benefits is obtained. The advantage for the left/right precues apparently arises from their association with the salient left/right spatial feature of the linear stimulus and response arrangements.

Additional evidence for salient-features coding can be found in studies that use a set of four two-dimensional visual stimuli (e.g., O, o, z, Z) assigned to a horizontal row of four keypress responses. Reaction times (RTs) are faster if the assignment is such that letter identity distinguishes the two left and two right responses (e.g., a left- 
to-right assignment of OozZ) than if it does not (e.g., a left-to-right assignment of OzoZ; Proctor \& Reeve, 1985; Proctor, Reeve, Weeks, Dornier, \& Van Zandt, 1991). For this experiment, letter identity is the most salient feature of the stimulus set, as indicated by faster identity discriminations than size discriminations (Proctor \& Reeve, 1985). Hence, responding is fastest for assignments in which the salient letter-identity feature of the stimulus set corresponds with the salient left/right spatial feature of the response set.

Most of the findings in support of salient-features coding have been obtained using stimulus and response sets from the visual-spatial modality. However, evidence suggests that S-R translation is based on salient features for the auditory-verbal modality as well. Gordon and Meyer (1984) conducted a series of experiments using sets of consonant-vowel stimuli and responses that varied on two dimensions. Their most widely examined set was BUH, DUH, PUH, and TUH $\left(/ \mathrm{b} \Lambda /, / \mathrm{d} \Lambda /, / \mathrm{p} \Lambda /\right.$, and $/ \mathrm{t}_{\Lambda} /$, in phonemic notation), for which the stimuli can be distinguished in terms of the voicing (PUH and TUH are unvoiced; BUH and DUH are voiced) and place of articulation (bilabial for BUH and PUH; alveolar for DUH and TUH) of the consonants. The experiments showed that RT to speak one of the syllables in response to an auditory stimulus from the same set is a function of the assignment of the stimuli to the responses. Responding was fastest when the assigned spoken response was the same as the auditory stimulus (e.g., respond "PUH" to PUH). More important, responding was faster when the response consonant shared a salient voicing feature with the stimulus (e.g., respond "DUH" to BUH) than when it did not (e.g., respond "DUH" to PUH), demonstrating a compatibility effect similar to those obtained for the visual-spatial modality.

Thus, a range of $\mathrm{S}-\mathrm{R}$ compatibility phenomena within the visual-spatial and auditory-verbal modalities are consistent with the view that salient-features coding underlies general translation processes. A crucial implication of this view, which has yet to be tested, is that predictable compatibility effects should also be obtained when the stimulus set or the response set is from the visual-spatial modality and the other set is from the auditory-verbal modality. That is, because the relative compatibility of alternative S-R assignments is determined only by whether salient features of the respective sets correspond, it should be possible to predict cross-modal compatibility effects on the basis of the mapping of salient features of stimulus sets in one modality to those of response sets in the other.

The purpose of the present study was to determine whether such cross-modal compatibility effects do in fact occur. In Experiment 1, the speech stimulus set used by Gordon and Meyer (1984) was assigned to four keypress responses in a manner similar to the assignments used by Proctor and Reeve (1985) for the two-dimensional visual stimuli. In Experiment 2, the stimulus and response modalities were reversed, with a horizontal row of four spatial-location stimuli assigned to the speech responses. Finally, in Experiment 3, Proctor and Reeve's twodimensional visual stimulus set was assigned to Gordon and Meyer's speech response set. For all the experiments, faster responses were expected when the salient features of the stimulus and response sets corresponded than when they did not.

\section{EXPERIMENT 1}

When four visual stimuli that differ on two dimensions (letter identity and size) are assigned to a row of four equally spaced response keys, RTs are faster when the salient letter-identity feature of the stimulus set corresponds to the salient left/right feature of the response set than when it does not (Proctor \& Reeve, 1985; Proctor et al., 1991). According to the salient-features perspective, a similar relation should hold when a twodimensional set of auditory stimuli is assigned to the keypress responses. In Experiment 1 we tested this prediction with the stimulus set of Gordon and Meyer (1984), in which the vocal stimuli differ on a salient voicing feature as well as a less salient place-of-articulation feature.

\section{Method}

Subjects. Sixteen students enrolled in introductory psychology classes at Purdue University participated for credit toward a course requirement. Each subject was tested in two sessions on successive days.

Apparatus and Stimuli. Stimuli consisted of the consonant-vowel syllables BUH, DUH, PUH, and TUH, spoken in a male voice. The stimuli differ along two dimensions, voicing and place of articulation of the consonant. PUH and TUH are unvoiced, and BUH and DUH are voiced; DUH and TUH are alveolar, and BUH and PUH are bilabial. The stimuli were digitized in 8-bit samples at a sampling rate of $12500 \mathrm{~Hz}$, using a Covox Voice-Master Key analog-to-digital speech digitizer attached to an AT-style microcomputer. Stimuli were presented at a rate of $12500 \mathrm{~Hz}$ on an external speaker using a Covox "Speech Thing" digital-to-analog converter connected to the computer. Stimuli were edited to 375 -msec duration with the digitizer's software waveform editor and approximately equated in peak amplitude. The amplitude of each stimulus was approximately $76 \mathrm{~dB}(\mathrm{~A})$, as measured by a sound-level meter, at a listening distance of about $55 \mathrm{~cm}$. The computer was used both for timing the presentation of stimuli and for recording response latency and accuracy. The subjects responded by pressing one of the four central keys on the bottom row of the computer's keyboard (the keys $\mathrm{V}$, $B, N$, and $M$ ) with the left middle, left index, right index, and right middle fingers, respectively.

To establish that voicing was a salient feature for the specific stimuli used in this experiment, a two-choice reaction task was conducted using two types of assignments. For one type, the stimuli with voiced consonants (BUH, DUH) were assigned to one response, and those with unvoiced consonants (PUH, TUH) were assigned to the other. For the other assignment type, the alveolar stimuli (DUH, TUH) were assigned to one response, and the bilabial stimuli (BUH, PUH) were assigned to the other. Twenty-four subjects were tested with each assignment type, performing 30 practice trials and 280 test trials. Responses were reliably faster when voicing distinguished the two responses $(M=702 \mathrm{msec})$ than when place did $\left[M=804 \mathrm{msec} ; F(1,46)=8.06, M S_{\mathrm{e}}=62,724, p<.01\right]$, and also tended, though not quite significantly, to be more accurate [percentages of errors (PEs) $=1.8 \%$ and $2.9 \%$, respectively, $F(1,46)=3.19, p=.08]$. Thus, the distinction between the voiced and unvoiced stimuli was salient for the stimuli used in this experiment. Moreover, there was no apparent difference in the relative ease of perceptibility of the voiced and unvoiced stimulus sets, be- 
cause the mean RTs for the voiced stimuli ( $M=754 \mathrm{msec})$ and the unvoiced stimuli ( $M=752 \mathrm{msec}$ ), collapsed across assignments, were equivalent.

Procedure. Each subject participated in two sessions that differed in terms of the type of S-R assignment used. Both types of assignments had the nonsalient place-of-articulation feature mapped to the two inner and two outer response locations, and so did not differ in this regard. The key distinction between the two assignment types was that the voicing feature corresponded to the salient left/right distinction of the response set (e.g., a left-to-right assignment of BUH, DUH, PUH, TUH) for one type of assignment, whereas for the other it was assigned to alternate locations (e.g., a left-toright assignment of PUH, DUH, TUH, BUH). The four mappings of specific stimuli to responses within each assignment type that met the constraints mentioned above were used equally often across subjects (the specific mappings were PUH, TUH, DUH, BUH; DUH, BUH, PUH, TUH; TUH, PUH, BUH, DUH; and BUH, DUH, TUH, PUH, for the former assignment type, and PUH, DUH, TUH, BUH; TUH, BUH, PUH, DUH; DUH, PUH, BUH, TUH; and BUH, TUH, DUH, PUH, for the latter). The order in which the subjects received the two types of assignment was counterbalanced, and the two specific mappings used by each subject had no individual S-R pairs in common.

Each session consisted of a 30-trial practice block and a 280-trial test block. Stimuli were presented randomly, with the restriction that each stimulus occurred an equal number of times within the first and second halves of the test block. Each auditory stimulus was presented $750 \mathrm{msec}$ after the response to the preceding stimulus. During the normal course of the experiment, the screen of the computer monitor was blank. If the subject made an error, the computer emitted a 500 -msec tone, and the sequence of trials was interrupted. The transcribed stimuli then were displayed on the screen, along with their assigned responses.

The subjects were instructed about the task and were allowed to study the response assignments before beginning. They were told to respond as quickly as possible while maintaining accuracy. An experimenter remained in the room during the first several practice trials to ensure that the subject understood the task. The RTs were recorded from the onset of the stimulus to the depression of the response key, with trials having times less than $200 \mathrm{msec}$ or greater than $1.5 \mathrm{sec}$ excluded. In this experiment, and in all subsequent experiments, the cutoffs were set to exclude approximately $2 \%$ of the responses. Trials on which an error was made were excluded from the RT data.

\section{Results and Discussion}

Mean RTs for correct responses and PEs were determined for each subject as a function of assignment type. Responses were faster for the assignment type in which voicing distinguished the two left and two right locations $(M=785 \mathrm{msec})$ than for the type in which it did not $\left[M=818 \mathrm{msec} ; F(1,15)=5.63, M S_{\mathrm{e}}=1,637, p<\right.$ $.05]$. Also, fewer errors tended to be made with the former assignment type $(\mathrm{PE}=2.1 \%)$ than with the latter $(\mathrm{PE}=$ $2.7 \%$ ), although this trend was not significant $[F(1,15)=$ $\left.2.87, M S_{\mathrm{e}}=7.99, p>.10\right]$. Thus, as predicted, responses were faster when the salient voicing feature of the stimulus set corresponded to the salient left/right feature of the response set than when it did not.

The obtained compatibility effect is consistent with the view that translation processes are based on salient features. Because the compatibility effect obtained with speech stimuli in Experiment 1 is similar to the effect obtained with visual stimuli in other studies (e.g., Proctor \& Reeve, 1985), it appears that the selection of keypress responses to speech stimuli is influenced by structural and assignment factors of the same type that affect such responses to visual stimuli.

\section{EXPERIMENT 2}

The presence of a compatibility effect for speech stimuli and spatial-location responses in Experiment 1 suggests a critical test for salient-features coding. If a compatibility effect is obtained with one S-R ensemble, an effect should also be obtained when the stimulus and response sets are reversed, as long as the relative salience of the features is maintained (see Weeks \& Proctor, 1990). Thus, in Experiment 2, we used spatial-location stimuli (four plus signs arranged in a row) assigned to vocal consonant-vowel responses (the spoken syllables "BUH," "DUH," "PUH,', and "TUH"). With this procedure, the left/right spatial feature now characterizes the stimulus set and the voiced/unvoiced feature characterizes the response set. Because the salient-feature structure from Experiment 1 is maintained, RTs should follow the same pattern. That is, they should be faster when the left/right feature of the stimulus set and the voicing feature of the response set correspond than when they do not.

\section{Method}

Subjects. Sixteen new students from the same population as in Experiment 1 served as subjects. Each subject participated in two sessions.

Apparatus, Stimuli, and Procedure. The visual stimuli were displayed in white at $99 \mathrm{~cd} / \mathrm{m}^{2}$ intensity on the dark background of a computer monitor. Viewing distance was approximately $50 \mathrm{~cm}$. Four equally spaced plus signs in a row designated the spatial locations and served as a warning signal. The target was a single plus sign that appeared directly beneath one of the plus signs in the row. Each plus sign subtended a visual angle of approximately $0.28^{\circ}$, with the row of four being approximately $2.4^{\circ}$ wide. The separation between the warning row and the target was approximately $0.60^{\circ}$.

The subjects responded with one of the four consonant-vowel syllables, “BUH," “DUH," "'PUH,” or “TUH." Response latencies were recorded with a voice-activated relay (Lafayette Instruments) attached to the computer. An experimenter remained in the room during the entire experiment to record errors and provide feedback to the subject.

The assignment types were identical to those in Experiment 1, except that the stimulus and response sets were reversed. For one type of assignment, the left/right stimulus distinction corresponded to the voicing distinction of the response set (e.g., left-to-right assignment of stimulus locations to "BUH," "DUH," "PUH," and "TUH"). For the other type of assignment, the left/right and voicing distinctions did not correspond (e.g., left-to-right assignment of stimulus locations to "PUH," "DUH," "TUH," and "BUH"). Stimuli and assignments were counterbalanced as in Experiment 1, and the number of trials was the same. A trial consisted of the presentation of the row of plus signs alone for $750 \mathrm{msec}$, followed by the presentation of the target. The row stayed on the screen while the target was present, and the entire display remained in view until a response was made. Trials were separated by a 2 -sec intertrial interval.

The subjects were given verbal instructions and allowed to study the $\mathrm{S}-\mathrm{R}$ assignments before beginning. In addition, they were told to respond as quickly as possible while maintaining accuracy. If 
the subject made an error, the experimenter told the subject the correct response. The $\mathrm{RTs}$ were recorded from the onset of the target stimulus, and only times from correct responses were included in the RT analyses. Trials having RTs less than $200 \mathrm{msec}$ or greater than $2 \mathrm{sec}$ were eliminated.

\section{Results and Discussion}

Responses were faster when the salient left/right spatial feature of the stimulus set corresponded to the salient voicing feature of the response set $(M=832 \mathrm{msec})$ than when it did not $[M=866 \mathrm{msec} ; F(1,15)=5.47$, $\left.M S_{\mathrm{e}}=1,710, p<.05\right]$. No difference between assignments was apparent in the error rates (PEs $=2.6 \%$ and $2.5 \%$, respectively, $F<1.0$ ). Thus, a compatibility effect was evident for visual location stimuli assigned to speech responses, with responses faster when salient features of the respective sets corresponded.

Experiments 1 and 2 together demonstrate that compatibility effects can be obtained across the visual-spatial and auditory-verbal modalities as a function of the correspondence of salient features of the stimulus and response sets. In Experiment 1, assignments for which the salient voicing feature of the speech stimuli corresponded to the salient left/right feature of the response set were more compatible than assignments for which it did not. Likewise, in Experiment 2, assignments for which the salient left/right feature of the stimulus set corresponded to the salient voicing feature of the speech response set were more compatible than assignments for which it did not. These results suggest that whether particular features are incorporated in either the stimulus or the response set does not alter the pattern of compatibility effects. Responses are faster when salient features correspond, even when the stimulus and response sets involve different modalities.

\section{EXPERIMENT 3}

For Experiments 1 and 2, the stimulus or response set from the visual-spatial modality was spatially arranged. However, cross-modal compatibility effects should not depend on the salient visual feature's being spatial, because compatibility effects within the visual-spatial modality also occur for stimulus sets for which the distinguishing feature is nonspatial (Proctor \& Reeve, 1985). In other words, compatibility effects should be found for any structured set of visual stimuli mapped to a structured set of vocal responses.

Gordon and Meyer (1984) reported an experiment that on the surface seems to contradict this prediction. As a control condition to one of their experiments using speech stimuli and responses, the syllables BUH, DUH, PUH, and TUH were presented visually. Unlike the condition in which the syllables were presented auditorily, no compatibility effect was obtained with the visual stimuli. However, although the set of syllables used by Gordon and Meyer contained a salient phonetic feature when presented auditorily, the transcribed set did not have a salient visual feature. Thus, a compatibility effect would be expected only if the visual stimuli were recoded phonetically, which apparently was not the case. According to the logic of salient-features coding, a compatibility effect should occur when a symbolic, visual stimulus set with a salient-feature structure is assigned to a structured vocal response set.

In Experiment 3, we tested for the presence of such a compatibility effect by assigning the two-dimensional set of visual, symbolic stimuli $(\mathrm{O}, \mathrm{o}, \mathrm{z}, \mathrm{Z})$, for which letter identity is salient (Proctor \& Reeve, 1985), to the twodimensional vocal response set ("BUH," "DUH,", "PUH," "TUH"), for which voicing is salient. Keeping the relation between the nonsalient place-of-articulation and lettersize features constant, performance for assignment types in which the salient features corresponded was compared with that for assignment types in which there was no correspondence between salient features of the sets. From the view that translation is facilitated when salient features correspond relative to when they do not, it was predicted that responses would be faster when the salient letter-identity feature of the stimulus set was in correspondence to the salient voicing feature of the response set than when it was not.

\section{Method}

Subjects. Sixteen new students from the population tested in the previous experiments served as subjects. Each subject participated in two sessions.

Apparatus, Stimuli, and Procedure. The apparatus was the same as in Experiment 2. An asterisk measuring $2.5 \mathrm{~mm}$ square was presented in the center of the screen as a warning signal. The target, which was one of the four letters $\mathrm{O}, \mathrm{o}, \mathrm{z}$, or $\mathrm{Z}$, appeared directly beneath the asterisk. The large letters subtended visual angles of approximately $0.57^{\circ}$ high $\times 0.28^{\circ}$ wide, and the small letters were $0.40^{\circ} \times 0.28^{\circ}$. The height of the entire stimulus display was approximately $1.15^{\circ}$. The subjects responded vocally with one of the four consonant-vowel syllables "BUH," "DUH," "PUH," or "TUH."

Two assignment types were used. For both types, the relation between the less salient features (size and place of articulation, respectively) was held constant. For one type, the letter-identity feature of the stimulus set corresponded to the voicing feature of the response set (e.g., O, o, z, Z assigned to "BUH," "DUH," "PUH," "TUH"). For the other, letter identity and voicing did not correspond (e.g., O, z, o, Z assigned to "PUH," "TUH," "DUH," "BUH"). Counterbalancing was performed as in Experiments 1 and 2.

A trial consisted of the presentation of the warning signal, followed after $750 \mathrm{msec}$ by the target. The warning signal and target remained in view until the subject made a response. Trials were separated by an interval of 2 sec.

The subjects were given verbal instructions and allowed to study the $\mathrm{S}-\mathrm{R}$ assignment before beginning. In addition, a card showing the assignment was provided. They were told not to depend on the assignment card, but to refer to it if necessary. The subjects were instructed to respond as quickly as possible while maintaining accuracy. Feedback was provided by the experimenter if an error was made. Trials having RTs less than $200 \mathrm{msec}$ or greater than $2.5 \mathrm{sec}$ were excluded.

\section{Results and Discussion}

An analysis of variance performed on the RT data showed an effect of assignment $[F(1,15)=5.26$, $\left.M S_{\mathrm{e}}=1,895, p<.05\right]$. Responses were faster for the assignment in which the letter-identity and voicing features corresponded ( $M=1,007 \mathrm{msec})$ than for the assign- 
ment in which they did not $(M=1,048 \mathrm{msec})$. The proportion of errors did not differ significantly for the two assignment types (PEs $=3.1 \%$ and $3.6 \%$, respectively, $F<1.0$ ). Thus, a compatibility effect based on the correspondence of the salient features was obtained.

Gordon and Meyer (1984) obtained a compatibility effect when speech stimuli were assigned to speech responses, but not when their visual equivalents were used. However, their visual stimulus set did not have a salient visual feature that dichotomized the alternatives. Experiment 3 shows that a compatibility effect can be obtained when a visual, symbolic stimulus set is assigned to speech responses, if the set has a salient-feature structure.

\section{GENERAL DISCUSSION}

There is considerable evidence that many S-R compatibility effects reflect the use of salient-features coding to translate between stimuli and responses (see, e.g., Proctor et al., 1990). Translation is faster when salient features of the sets correspond than when they do not. Previous research has shown salient-features coding to hold when both the stimulus and response sets come from within either the visual-spatial modality or the auditoryverbal modality. In the present experiments, we tested the implication that cross-modal compatibility effects that are predictable from the salient-feature structure of the stimulus and response sets should also occur.

In Experiment 1, a speech stimulus set (BUH, DUH, PUH, TUH \} used by Gordon and Meyer (1984) was assigned to a row of four keypress responses. Responses were faster when the salient voicing feature of the stimulus set corresponded to the salient left/right feature of the response set than when it did not. In Experiment 2, the stimulus and response sets were reversed, so that a row of four stimulus locations was assigned to speech responses, and a similar compatibility effect again was obtained. These experiments together demonstrate that it does not matter which of the salient features, the voicing feature of the speech syllables or the left/right feature of the row of locations, applies to the stimulus set or the response set. The critical factor is whether the features correspond.

Gordon and Meyer (1984) previously failed to find a compatibility effect when visual, symbolic stimuli were assigned to speech responses. However, their stimulus set lacked a salient visual feature. Experiment 3 showed that a compatibility effect occurs when a set of visually presented symbolic stimuli with a salient-feature structure is assigned to the speech response set. This result complements Gordon and Meyer's finding that no such compatibility effect is obtained when the visual stimulus set lacks structure. It further illustrates that neither the modalities of the stimulus and response sets nor the specific features of the set members need be identical.

The present findings join others in showing that $S-R$ compatibility relations generalize across speech and nonspeech stimuli and responses. For example, Inhoff, Rosenbaum, Gordon, and Campbell (1984), and Rosenbaum, Gordon, Stillings, and Feinstein (1987) showed similar compatibility effects for sequences of keypresses and sequences of vocal responses. Inhoff et al. examined twochoice RT for mirror-image keypress sequences of varying lengths made with the index, middle, and ring fingers of the left and right hands. The signal to respond was a left- or right-location visual stimulus mapped either compatibly (e.g., left location signaled the left-hand sequence) or incompatibly (e.g., left location signaled the right-hand sequence) to the response sequences. Mean RT increased with sequence length and was faster when the S-R assignment was compatible than when it was not, with the two effects being additive. Subsequent experiments that used heterogeneous sequences (response sequences beginning with one hand and ending with the other) showed a reduced compatibility effect relative to homogeneous sequences (response sequences performed with one hand), indicating that whereas the direction of the compatibility effect is determined by the initial keypress in a sequence, $\mathrm{RT}$ is also influenced by the relation between the signal to respond and noninitial keypresses.

Rosenbaum et al. (1987) performed the complement to this work by conducting similar experiments with vocal response sequences composed from the sets $\{/ \mathrm{gi} /, / \mathrm{bi} /$, $/ \mathrm{di} /\}$ and $\{/ \mathrm{gu} /, / \mathrm{bu} /, / \mathrm{du} /\}$, and either a high- or lowpitched tone signal to respond. The higher pitched /i/ sound is compatible with the high-pitched tone signal and the lower pitched $/ \mathrm{u} /$ sound is compatible with the lowpitched tone signal. As was found for keypress responses, RT increased with sequence length and was faster when the signal to respond was compatible with the response sequence. Moreover, comparisons between heterogeneous (e.g., /bibu/) and homogeneous (e.g., /bibi/) sequences also showed a reduced compatibility effect for the heterogeneous sequences.

The similarity of the results obtained by Inhoff et al. (1984) with keypress response sequences and by Rosenbaum et al. (1987) with vocal response sequences suggests that response selection is performed similarly for the two response sets. Also, the finding that a compatibility effect occurs for the assignment of a nonspeech auditory stimulus (tone pitch) to a speech response is consistent with the implication of our experiments that S-R compatibility effects obtained with speech stimuli and/or responses are not restricted to processes operating within a speech module.

There is general agreement that most $S-R$ compatibility effects are a function of the coding of stimulus and response sets and the resultant complexity of translation between them (Proctor \& Reeve, 1990). The specific compatibility effects that are observed depend on stimulus and response set structure and the degree to which the salient features correspond. As illustrated by the present experiments, the effects are not restricted to stimulus and response sets that share a common feature. Rather, the primary determinant of compatibility is whether the mapping of the respective sets is such that features correspond.

For situations in which stimulus features would appear to have a direct relation to response features, it is tempting to attribute any resulting compatibility effect to links 
between the perceptual and motor systems. In the case of speech compatibility effects, the obvious similarity of features of the speech stimuli and responses led to earlier interpretations of the compatibility effect in terms of a privileged relation between hearing and producing speech (e.g., Gordon, 1990). Yet, our findings show that similar compatibility effects can be obtained when the features or modalities of the stimulus and response sets are not the same, implicating general translation processes. In another recent case, Michaels (1988) found a compatibility effect between the "destination" of a stimulus with apparent movement characteristics and a "catching" response (forward movement of a joystick). She attributed this compatibility effect to the direct affordance of a catching action at the destination. However, subsequent evidence has implicated more general translation processes as the basis for this effect (Proctor, Van Zandt, Lu, \& Weeks, 1993). As with the relation between speech stimuli and responses, the research with apparently moving stimuli showed that compatibility does not depend on a direct, special relation between the stimuli and the responses.

Given that the evidence indicates that most S-R compatibility effects are due to general translation processes mediating between stimuli and responses, we argue that it is necessary to consider the role of translation in any newly discovered compatibility effect. Because translation processes are the major factor determining initial performance in most choice-reaction tasks (e.g., Welford, 1976) and may have enduring effects (Dutta \& Proctor, 1992), any new S-R compatibility effects likely will have a translation basis. Models that attribute S-R compatibility effects to direct interactions between the perceptual and motor systems, without a mediating translation stage, may not provide complete explanations of these effects.

\section{REFERENCES}

Cauraugh, J. H., \& Horrell, J. F. (1989). Advance preparation of discrete motor responses: Nonmotoric evidence. Acta Psychologica, 72, 117-138.

DutTa, A., \& Proctor, R. W. (1992). Persistence of stimulus-response compatibility effects with extended practice. Journal of Experimental Psychology: Learning, Memory, \& Cognition, 18, 801-809.

GoRDON, P. C. (1990). Perceptual-motor processing in speech. In R. W. Proctor \& T. G. Reeve (Eds.), Stimulus-response compatibility: An integrated perspective (pp. 343-362). Amsterdam: North-Holland. GoRDon, P. C., \& MEYER, D. E. (1984). Perceptual-motor processing of phonetic features in speech. Journal of Experimental Psychology: Human Perception \& Performance, 10, 153-178.
Hasbrouce, T., Guiard, Y., \& Ottomani, L. (1990). Principles of response determination: The list-rule model of SR compatibility. Bulletin of the Psychonomic Society, 28, 327-330.

Inhoff, A. W., Rosenbaum, D. A., Gordon, A. M., \& Campbell, J. A. (1984). Stimulus-response compatibility and motor programming of manual response sequences. Journal of Experimental Psychology: Human Perception \& Performance, 10, 724-733.

Michaels, C. F. (1988). S-R compatibility between response position and destination of apparent motion: Evidence of the detection of affordances. Journal of Experimental Psychology: Human Perception \& Performance, 14, 231-240.

Miller, J. (1982). Discrete versus continuous stage models of human information processing: In search of partial output. Joumal of Experimental Psychology: Human Perception \& Performance, 8, 273-296.

Proctor, R. W., \& ReEve, T. G. (1985). Compatibility effects in the assignment of symbolic stimuli to discrete finger responses. Journal of Experimental Psychology: Human Perception \& Performance, 11 , 623-639.

Proctor, R. W., \& ReEve, T. G. (Eds.) (1990). Stimulus-response compatibility: An integrated perspective. Amsterdam: North-Holland.

Proctor, R. W., Reeve, T. G., \& Weeks, D. J. (1990). A triphasic approach to the acquisition of response-selection skill. In G. H. Bower (Ed.), The psychology of learning and motivation (Vol. 26, pp. 207240). San Diego, CA: Academic Press.

Proctor, R. W., Reeve, T. G., Weeks, D. J., Dornier, L., \& VAN ZANDT, T. (1991). Acquisition, retention, and transfer of response-selection skill in choice-reaction tasks. Journal of Experimental Psychology: Learning, Memory, \& Cognition, 17, 497-507.

Proctor, R. W., VAN ZandT, T., LU, C.-H., \& Weeks, D. J. (1993). Stimulus-response compatibility for moving stimuli: Perception of affordances or directional coding? Journal of Experimental Psychology: Human Perception \& Performance, 19, 81-91.

Reeve, T. G., \& Proctor, R. W. (1984). On the advance preparation of discrete finger responses. Journal of Experimental Psychology: $\mathrm{Hu}_{\text {- }}$ man Perception \& Performance, 10, 541-553.

ReEve, T. G., \& Proctor, R. W. (1990). The salient-features coding principle for spatial- and symbolic-compatibility effects. In $\mathbf{R}$. W. Proctor \& T. G. Reeve (Eds.), Stimulus-response compatibility: An integrated perspective (pp. 163-180). Amsterdam: North-Holland.

Rosenbaum, D. A., Gordon, A. M., Stillings, N. A., \& Feinstein, M. H. (1987). Stimulus-response compatibility in the programming of speech. Memory \& Cognition, 15, 217-224.

UMiltA, C., \& Nicoletri, R. (1990). Spatial stimulus-response compatibility. In R. W. Proctor \& T. G. Reeve (Eds.), Stimulus-response compatibility: An integrated perspective (pp. 89-116). Amsterdam: North-Holland.

Weeks, D. J., \& Proctor, R. W. (1990). Salient-features coding in the translation between orthogonal stimulus and response dimensions. Journal of Experimental Psychology: General, 119, 355-366.

WELFoRD, A. T. (1976). Skilled performance: Perceptual and motor skills. Glenview, IL: Scott, Foresman.

(Manuscript received February 10, 1993; revision accepted for publication March 23, 1993.) 